University of Nebraska - Lincoln

DigitalCommons@University of Nebraska - Lincoln

$10-30-2014$

Dimensional structure of DSM-5 posttraumatic stress symptoms:

Support for a hybrid Anhedonia and Externalizing Behaviors

model

Cherie Armour

Jack Tsai

Tory A. Durham

Ruby Charak

Tracey L. Biehn

See next page for additional authors

Follow this and additional works at: https://digitalcommons.unl.edu/psychfacpub

Part of the Psychology Commons

This Article is brought to you for free and open access by the Psychology, Department of at

DigitalCommons@University of Nebraska - Lincoln. It has been accepted for inclusion in Faculty Publications, Department of Psychology by an authorized administrator of DigitalCommons@University of Nebraska - Lincoln. 


\section{Authors}

Cherie Armour, Jack Tsai, Tory A. Durham, Ruby Charak, Tracey L. Biehn, Jon D. Elhai, and Robert H. Pietrzak 


\title{
Dimensional structure of DSM-5 posttraumatic stress symptoms: Support for a hybrid Anhedonia and Externalizing Behaviors model
}

\author{
Cherie Armour ${ }^{\mathrm{a}, ~}{ }^{*}$, Jack Tsai ${ }^{\mathrm{b}, \mathrm{c}}$, Tory A. Durham ${ }^{\mathrm{d}}$, Ruby Charak ${ }^{\mathrm{e}}$, Tracey L. Biehn ${ }^{\mathrm{d}}$, \\ Jon D. Elhai ${ }^{\mathrm{d}, \mathrm{f}}$, Robert H. Pietrzak ${ }^{\mathrm{c}, \mathrm{g}}$ \\ a Psychology Research Institute, University of Ulster, Coleraine, Northern Ireland, UK \\ ${ }^{\mathrm{b}}$ United States Department of Veterans Affairs New England Mental Illness Research, Education, and Clinical Center, West Haven, CT, USA \\ ${ }^{\mathrm{c}}$ Department of Psychiatry, Yale University School of Medicine, New Haven, CT, USA \\ d Department of Psychology, University of Toledo, USA \\ e Department of Psychology, University of Nebraska-Lincoln, Lincoln, NE, USA \\ ${ }^{\mathrm{f}}$ Department of Psychiatry, University of Toledo, USA \\ ${ }^{g}$ United States Department of Veterans Affairs National Center for Posttraumatic Stress Disorder, Clinical Neurosciences Division, VA Connecticut Healthcare \\ System, West Haven, CT, USA
}

\section{A R T I C L E I N F O}

\section{Article history:}

Received 23 July 2014

Received in revised form

1 October 2014

Accepted 30 October 2014

Keywords:

Posttraumatic stress disorder

DSM-5

7-Factor hybrid model

Confirmatory factor analysis

Latent structure

\begin{abstract}
A B S T R A C T
Several revisions to the symptom clusters of posttraumatic stress disorder (PTSD) have been made in the 5th edition of the Diagnostic and Statistical Manual of Mental Disorders (DSM-5). Central to the focus of this study was the revision of PTSD's tripartite structure in DSM-IV into four symptom clusters in DSM-5. Emerging confirmatory factor analytic (CFA) studies have suggested that DSM-5 PTSD symptoms may be best represented by one of two 6-factor models: (1) an Externalizing Behaviors model characterized by a factor which combines the irritability/anger and self-destructive/reckless behavior items; and (2) an Anhedonia model characterized by items of loss of interest, detachment, and restricted affect. The current study conducted CFAs of DSM-5 PTSD symptoms assessed using the PTSD Checklist for DSM-5 (PCL5 ) in two independent and diverse trauma-exposed samples of a nationally representative sample of 1484 U.S. veterans and a sample of 497 Midwestern U.S. university undergraduate students. Relative fits of the DSM-5 model, the DSM-5 Dysphoria model, the DSM-5 Dysphoric Arousal model, the two 6-factor models, and a newly proposed 7-factor Hybrid model, which consolidates the two 6-factor models, were evaluated. Results revealed that, in both samples, both 6-factor models provided significantly better fit than the 4-factor DSM-5 model, the DSM-5 Dysphoria model and the DSM-5 Dysphoric Arousal model. Further, the 7-factor Hybrid model, which incorporates key features of both 6-factor models and is comprised of re-experiencing, avoidance, negative affect, anhedonia, externalizing behaviors, and anxious and dysphoric arousal symptom clusters, provided superior fit to the data in both samples. Results are discussed in light of theoretical and empirical support for the latent structure of DSM-5 PTSD symptoms.
\end{abstract}

() 2014 Elsevier Ltd. All rights reserved.

\section{Introduction}

There has been longstanding debate within the academic literature with regard to the underlying dimensionality of Posttraumatic Stress Disorder (PTSD) (cf. Yufik and Simms, 2010). The

\footnotetext{
* Corresponding author. Psychology Research Institute, University of Ulster, Coleraine Campus, Cromore Road, Coleraine, Northern Ireland, BT52 1SA, UK. Tel.: +44 (0) 2870123374

E-mail address: armour.cherie@gmail.com (C. Armour).
}

most recent, 5th edition of the DSM (DSM-5; American Psychiatric Association, 2014), characterizes PTSD as a heterogenous disorder comprised of four symptom clusters which supersedes the tripartite model utilized by the DSM from 1980 to 2013. A body of confirmatory factor analytic (CFA) studies found that the dimensional structure of DSM-based PTSD symptoms, as represented in the DSM-IV (APA, 1994) through to the DSM-IV-TR (APA, 2000), are best represented by four rather than three symptom clusters (King et al., 1998; Simms et al., 2002). Indeed, two four-factor models of PTSD-the Emotional Numbing model and the Dysphoria model-have been proposed and supported across a variety of trauma- 
exposed populations from a variety of cultural backgrounds and utilizing different assessment instruments (Elhai and Palmieri, 2011; Gootzeit and Markon, 2011; Yufik and Simms, 2010). The recent proposal of two 6-factor DSM-5 models-the Anhedonia model and the Externalizing Behavior model (Liu et al., 2014; Tsai et al., in press, respectively)-is reminiscent of earlier debates surrounding the two 4-factor DSM-IV/DSM-IV-TR models. The current study focuses on the assessment of these models in comparison to a 7-factor Hybrid model, which combines key features of both 6 -factor models, in order to determine which model better represents the latent structure of DSM-5 PTSD symptoms. This is an important line of research, as it can help shed light on diagnosis, treatment planning, and elucidating the underlying nature of PTSD.

The debate surrounding which one of the two 4-factor DSM-IVTR models-the Emotional Numbing model (King et al., 1998; reexperiencing, avoidance, numbing, hyperarousal) or the Dysphoria model (Simms et al., 2002; re-experiencing, avoidance, dysphoria, hyperarousal)-best represented the dimensional structure of PTSD was never fully resolved. The Emotional Numbing model was conceived from theoretical and empirical support that Numbing and Avoidance were two distinct factors (Asmundson, Stapleton, \& Taylor, 2004), whereas the Dysphoria model was conceived on proposals that certain PTSD items, the dysphoric items, were less specific to the overall PTSD construct (cf. Watson, 2009). Of note, a meta-analytic review of 40 CFA studies concluded that the Dysphoria model provided modestly better fit to the pooled data compared to the Emotional Numbing model (Yufik and Simms, 2010). Given marginal differences in fit, however, it is entirely plausible that a different selection of PTSD studies could have resulted in better fit being demonstrated by the Emotional Numbing model. Ultimately, the two four-factor models differed in their assignment of three PTSD symptoms-sleep difficulties, anger/irritability, and difficulty concentrating, which may not be unique indicators of specific factors (Shevlin et al., 2009).

The most recent development in the study of the dimensional structure of DSM-IV-TR PTSD symptoms (APA, 2000) was the proposal of the Dysphoric Arousal model, which posits a 5-factor model of re-experiencing, avoidance, numbing, dysphoric arousal, and anxious arousal symptoms (Elhai et al., 2011). The key difference from preceding four-factor models was the separation of hyperarousal into two distinct clusters of dysphoric arousal and anxious arousal. This was based on Watson's (2005) proposal that items representing sleep disturbance, irritability, and difficulty concentrating involve general distress and/or dysphoria which is characteristic of depressive disorders, whereas remaining hyperarousal items of hypervigilance and exaggerated startle are conceptually different and more characteristic of fear-based disorders (Elhai et al., 2011). This model quickly gathered empirical support across a variety of samples, which differed with respect to trauma exposure, life stage (adolescence or adulthood), culture, and PTSD assessment instrument (Armour et al., 2013a, 2013b, 2012,; Contractor et al., 2013; Pietrzak et al., 2012; Reddy et al., 2013; Wang et al., 2013). The five factors that comprise the Dysphoric Arousal model have additionally been shown to differentially relate to health and neurobiological variables (HarpazRotem et al., 2014, Pietrzak et al., 2013a, 2013b).

The proposal and developing evidence base of the Dysphoric Arousal model coincided with the release of DSM-5 (APA, 2014). Given that the DSM-5 was published in May 2013, it was preceded by the wealth of empirical evidence supporting either the Emotional Numbing model or the Dysphoria model. The Dysphoric Arousal model had likely not gained sufficient momentum to be considered for DSM-5. Thus, it was no surprise that the DSM-5 criteria for PTSD included a 4-factor PTSD model. In this model, however, a number of the existing symptoms were revised and three additional symptoms were added. Therefore, the 4-factor model of PTSD in the DSM-5 is not a direct replication of the Emotional Numbing or Dysphoria model. Of note, this model most closely resembles the Emotional Numbing model. Specifically, the DSM-5 model of PTSD includes re-experiencing (RE), avoidance (AV), negative alterations in cognitions and mood (NACM; similar to the numbing model's numbing factor), and alterations in arousal and reactivity (AR; similar to the numbing model's hyperarousal factor) symptom clusters (Friedman et al., 2011).

Since the publication of the DSM-5, several researchers have investigated the fit of the DSM-5 4-factor model in data gleaned from a national sample of U.S. adults, a clinical sample of U.S. veterans, undergraduate psychology students, and primary care patients (Armour et al., 2014; Biehn et al., 2013; Contractor et al., 2014; Elhai et al., 2012; Miller et al., 2013). The general trend in these studies has been to compare the DSM-5 model to a modified DSM-5 model, which more closely represents the DSM-IV-TR Dysphoria model. To date, the DSM-5 model has received the most support; however, Miller et al. (2013) found support for a DSM-5 Dysphoria model in their national sample of U.S. adults and clinical sample of veterans. Thus, these findings mirror those of the two $D S M-I V-T R$ 4-factor models in that neither model is conclusively preferential.

Recently, two separate research teams simultaneously published new DSM-5 PTSD models comprised of 6-factors (Liu et al., 2014; Tsai et al., in press). Liu et al. (2014) assessed PTSD's latent structure using data from Chinese earthquake survivors $(N=1196)$ who were assessed for PTSD using the PTSD checklist for DSM-5 (PCL-5). In total, six competing DSM-5 models were assessed, including the DSM-5 four-factor model, a Dysphoria version of this model, a Dysphoric Arousal version of the DSM-5 model, and a newly proposed 6-factor Anhedonia model. The latter model incorporated both the separation of hyperarousal as per the Dysphoric Arousal model, as well as the separation of the new NACM factor into two distinct constructs of 'negative alterations in cognitions and mood' and 'anhedonia'; representing positive and negative affect (Watson, 2005, 2009; Watson et al., 2011). The 6factor Anhedonia model was found to better fit these data compared to all assessed alternative models.

Tsai et al. (in press), assessed PTSD's latent structure using PCL-5 data from a nationally representative sample of U.S. veterans ( $N=1484$ ). Once again, these researchers aimed to build on the Dysphoric Arousal model by incorporating dysphoric arousal and anxious arousal factors whilst further evaluating the addition of a new "externalizing behaviors" factor comprised of irritable or aggressive behavior and self-destructive or reckless behavior (a new symptom added to PTSD's Criterion E in DSM-5). The symptoms in this factor are deemed externalizing given they assess selfinitiating aggressive behaviors which are thought to represent deficits in emotion regulation and impulse control (Freiedman, 2013; Roberton et al., 2012). These are distinct from other symptoms located in the E criterion which measure thoughts, feelings, and passive experiences. Three competing models were tested in this study: the DSM-5 model, the DSM-5 Dysphoria model, and a newly proposed 6-factor 'Externalizing Behaviors' model, and assessed in the total sample, a subsample of female veterans only, and a subsample of veterans with lifetime PTSD. Results revealed that the 6-factor Externalizing Behaviors model provided a better fit to the data compared to the two alternative models across all three samples. Symptom mappings of the DSM-5 (APA, 2014), Anhedonia (Liu et al., 2014), and Externalizing Behaviors (Tsai et al., in press) models are shown in Table 1.

The two 6-factors models are similar in that both group the same items together to represent re-experiencing, avoidance, and anxious arousal symptoms. However, whereas the Externalizing 
Table 1

Item mappings for the five PTSD models assessed in U.S. veterans and Midwestern students.

\begin{tabular}{|c|c|c|c|c|c|c|}
\hline DSM-5 symptom & DSM-5 & Dysphoria & Dysphoric arousal & Externalizing behaviors & Anhedonia & Hybrid \\
\hline 1. Intrusive thoughts & $\mathrm{R}$ & $\mathrm{R}$ & $\mathrm{R}$ & $\mathrm{R}$ & $\mathrm{R}$ & $\mathrm{R}$ \\
\hline 2. Nightmares & $\mathrm{R}$ & $\mathrm{R}$ & $\mathrm{R}$ & $\mathrm{R}$ & $\mathrm{R}$ & $\mathrm{R}$ \\
\hline 3. Flashbacks & $\mathrm{R}$ & $\mathrm{R}$ & $\mathrm{R}$ & $\mathrm{R}$ & $\mathrm{R}$ & $\mathrm{R}$ \\
\hline 4. Emotional cue reactivity & $\mathrm{R}$ & $\mathrm{R}$ & $\mathrm{R}$ & $\mathrm{R}$ & $\mathrm{R}$ & $\mathrm{R}$ \\
\hline 5. Physiological cue reactivity & $\mathrm{R}$ & $\mathrm{R}$ & $\mathrm{R}$ & $\mathrm{R}$ & $\mathrm{R}$ & $\mathrm{R}$ \\
\hline 6. Avoidance of thoughts & A & A & A & A & A & A \\
\hline 7. Avoidance of reminders & A & A & A & A & A & A \\
\hline 8. Trauma-related amnesia & NACM & $\mathrm{D}$ & NACM & NACM & NACM & NA \\
\hline 9. Negative beliefs & NACM & $\mathrm{D}$ & NACM & NACM & NACM & NA \\
\hline 10. Blame of self or others & NACM & $\mathrm{D}$ & NACM & NACM & NACM & NA \\
\hline 11. Negative trauma-related emotions & NACM & $\mathrm{D}$ & NACM & NACM & NACM & NA \\
\hline 12. Loss of interest & NACM & $\mathrm{D}$ & NACM & NACM & An & An \\
\hline 13. Detachment & NACM & $\mathrm{D}$ & NACM & NACM & An & An \\
\hline 14. Restricted affect & NACM & $\mathrm{D}$ & NACM & NACM & An & An \\
\hline 15. Irritability/anger & AR & $\mathrm{D}$ & DA & EB & DA & EB \\
\hline 16. Self-destructive/reckless behavior & AR & AR & DA & EB & DA & EB \\
\hline 17. Hypervigilance & AR & AR & $\mathrm{AA}$ & AA & AA & AA \\
\hline 18. Exaggerated startle response & AR & AR & $\mathrm{AA}$ & AA & AA & AA \\
\hline 19. Difficulty concentrating & AR & $\mathrm{D}$ & DA & DA & DA & DA \\
\hline 20. Sleep disturbance & AR & $\mathrm{D}$ & DA & DA & DA & DA \\
\hline
\end{tabular}

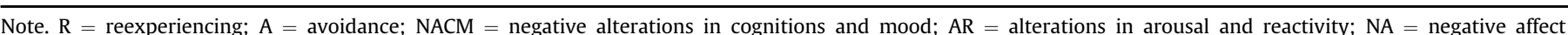
$\mathrm{An}=$ anhedonia; $\mathrm{EB}=$ externalizing behaviors; $\mathrm{AA}=$ anxious arousal; $\mathrm{DA}=$ dysphoric arousal.

model includes a NACM factor comprised of seven PTSD symptoms, the Anhedonia model proposes that this factor is comprised of only four PTSD items. The remaining three items are used to create an anhedonia factor. Similarly, the Anhedonia model suggest a dysphoric arousal factor comprised of four symptoms whereas the Externalizing Behaviors model proposes the inclusion of only two symptoms; the remaining two items comprise the externalizing behaviors factor.

In the current study, we propose a hybrid model that combines key features of both the Anhedonia and Externalizing 6-factor DSM-5 PTSD models, including RE, AV, negative affect, anhedonia, externalizing behavior, anxious arousal, and dysphoric arousal. As a result, the hybrid model is based on theoretical and empirical evidence which proposes that hyperarousal should consist of anxious and dysphoric arousal; that negative and positive affect are two distinct constructs; and that externalizing behaviors characterized by difficulties with emotion regulation and impulse control and may constitute a separate factor distinct from other PTSD symptoms representing passive experiences, thoughts, and feelings (Friedman, 2013; Steiner et al., 1997; Watson, 2005, 2009; Watson et al., 2011).

Models assessed herein were the current DSM-5 model, the DSM-5 Dysphoria model, the DSM-5 Dysphoric Arousal model, the Anhedonia model, the Externalizing Behaviors model, and a newly proposed 7-factor Hybrid model. We evaluated these models using CFAs in two diverse samples: a nationally representative U.S. veteran sample and a trauma-exposed U.S undergraduate student sample. We hypothesized that the 7-factor Hybrid model would evidence superior fit given that it combines a number of theoretically and empirically support revisions to PTSD's latent structure. This model can be viewed in Fig. 1.

\section{Methods}

\subsection{Participants}

\subsubsection{U.S. veterans}

The National Health and Resilience in Veterans Study (NHRVS) is a nationally representative survey of 1484 U.S. veterans that was conducted between September to October 2013. Participants completed a 60-min anonymous web-based survey. The NHRVS sample was drawn from a research panel of more than 80,000 households maintained by GfK Knowledge Networks, Inc., a survey research firm that owns and uses KnowledgePanel ${ }^{\circledR}$, a probabilitybased, online non-volunteer access survey panel of a nationally representative sample of U.S. adults that covers approximately $98 \%$ of U.S. households. To permit generalizability of study results to the entire population of U.S. veterans, poststratification weights were applied based on demographic distributions (i.e., age, gender, race/ ethnicity, education, Census region, and metropolitan area) from the most contemporaneous Current Population Survey (U.S. Census Bureau, 2010). All participants provided informed consent and the study was approved by the Human Subjects Subcommittee of the VA Connecticut Healthcare System and VA Office of Research \& Development.

\subsubsection{Midwestern university students}

The Midwestern university student sample was recruited from 2012 to 2014 via the University's Sona Systems recruitment website subsequent to IRB approval that was granted in fall 2013. Subjects were awarded course credit upon completion of their participation. Screening criteria were included to ensure that only subjects who have been exposed to a traumatic event were eligible to enroll in the study; a more thorough trauma exposure assessment was administered as part of the study (discussed below). After recruitment, subjects were directed to an informed consent statement and then the web survey.

\section{Assessments}

\subsection{Trauma history}

\subsubsection{U.S. veterans}

The Trauma History Screen (THS; Carlson et al., 2011) is a selfreport measure that assesses the lifetime occurrence of 14 potentially traumatic events. These events include early life events such as physical or sexual assault during childhood; as well as events that more commonly occur in adulthood, such as motor vehicle accident and unexpected loss of a loved one. An additional potentially traumatic event, life-threatening illness or injury item, was added to the THS in the NHRVS. Potentially traumatic events endorsed ranged from 0 to 15 . 

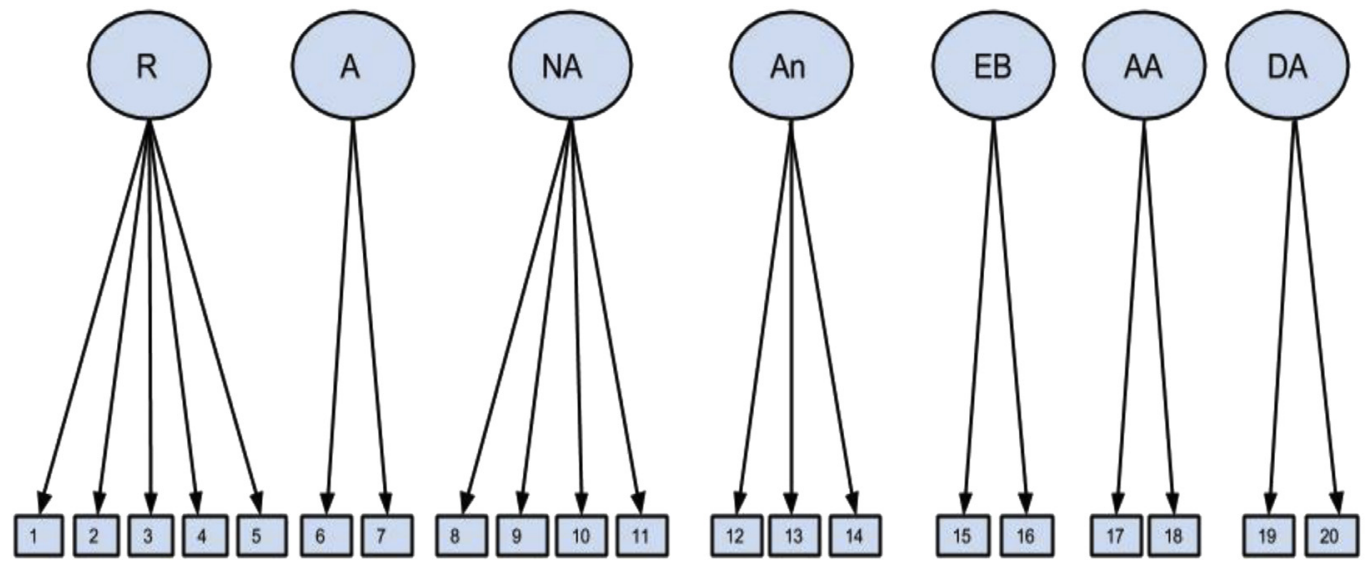

Note. $\mathrm{R}=$ Re-experiencing; $\mathrm{A}=$ Avoidance; $\mathrm{NA}=$ Negative affect $; \mathrm{An}=$ Anhedonia; $\mathrm{EB}=$ Externalizing Behaviors; $\mathrm{AA}=$ Anxious Arousal; DA = Dysphoric Arousal. 1. Intrusive thoughts; 2. Nightmares; 3. Flashbacks; 4. Emotional cue reactivity; 5. Physiological cue reactivity; 6 . Avoidance of thoughts; 7. Avoidance of reminders; 8 . Traumarelated amnesia; 9. Negative beliefs; 10 . Blame of self or others; 11. Negative trauma-related emotions; 12. Loss of interest; 13. Detachment; 14. Restricted affect; 15. Irritability/anger; 16. Self-destructive/reckless behavior; 17. Hypervigilance; 18. Exaggerated startle response; 19. Difficulty concentrating; 20. Sleep disturbance.

Intercorrelations of the latent factors are not shown but incorporated into confirmatory factor modeling.

Fig. 1. Path diagram representing item distribution across the 7-factors of the Hybrid model.

\subsubsection{Midwestern university students}

The Structured Clinical Interview for DSM-IV (SCID) PTSD trauma screen is a structured diagnostic interview to assess for several Axis I and Axis II disorders (First et al., 2002). The SCID PTSD trauma screen consists of a single question used to screen for potential trauma exposure. Only participants who endorsed trauma exposure on this screening question were eligible to participate (Elhai et al., 2008).

The Stressful Life Events Screening Questionnaire (SLESQ: Goodman et al., 1998) is a 13-item self-report measure assessing for lifetime exposure to traumatic events for DSM-IV PTSD. A modified version for DSM-5 was used to include a question inquiring about repeated or extreme exposure to gruesome or horrific details of trauma, and inquiry as to whether or not the trauma was experienced through electronic media or one's occupation. This was included to be consistent with DSM-5 PTSD criteria and has been used in a previous study (Elhai et al., 2012). If subjects endorsed more than one trauma, they were asked to elect their most upsetting trauma for later inquiry of PTSD symptoms. The SLESQ has demonstrated good test-retest reliability with a median kappa of .73 and good convergent and concurrent validity $(r=.77)$ with a longer, more extensive, interview of trauma exposure (Goodman et al., 1998).

\subsection{DSM-5 PTSD symptoms}

In both samples, PTSD was assessed using the PTSD Checklist for DSM-5 (PCL-5; Weathers et al., 2013), a self-report measure of DSM-5 PTSD symptoms. Participants were asked to report the amount of distress each symptom has caused them over the past month on a five-point Likert-type scale $(0=$ "Not at all" to 4 = "Extremely"), based on their most upsetting trauma elected from the THS or SLESQ. The PCL-5 demonstrated good internal consistency in both veteran (Cronbach's alpha $=.95$ ) and student (alpha $=.95$ ) samples. Test-retest reliability for the original PCL has been demonstrated to be quite high $(r=.96)$ in Vietnam veterans (reviewed in McDonald and Calhoun, 2010) and has found to be highly correlated with other self-report measures of PTSD (e.g., Mississippi Scale for Combat Related PTSD r's from .85 to .93) and clinician-administered interviews (e.g., Clinician-Administered PTSD Scale, $r=.79$ ) demonstrating good validity for the measure (reviewed in McDonald and Calhoun, 2010).

\subsection{Data screening and exclusion criteria}

\subsubsection{U.S. veterans}

A total of 1602 adults responded "Yes" to an initial screening question that confirmed veteran status and 1484 participated in the NHRVS, resulting in a response rate of $92.6 \%$. Panel members are recruited through national random samples, originally by telephone and now almost entirely by postal mail. Households are provided with access to the Internet and computer hardware if needed. KnowledgePanel ${ }^{\circledR}$ recruitment uses dual sampling frames that include both listed and unlisted telephone numbers, telephone and non-telephone households, and cell-phone-only households, as well as households with and without Internet access.

\subsubsection{Effective sample characteristics}

The effective sample $(n=1484)$ had a mean age of 60.4 $(S D=15.3)$ and ranged from 20 to 94 years old. The majority of subjects were male ( $n=1326$; weighted $89.7 \%)$ with at least some college education ( $n=1247$; weighted $67.0 \%$ ). The majority was Caucasian ( $n=1204$; weighted 75.4\%), African-American $(n=112$; weighted $9.7 \%$ ), or Hispanic ( $n=99$; weighted 9.1\%). Many were employed ( $n=583$; weighted $41.6 \%$ ) and most were married ( $n=1016$; weighted 64.8\%). In the study sample, the most endorsed traumatic events were "sudden death of close family member or friend" ( $n=885 ; 60.9 \%$ ), seeing someone die suddenly or get badly hurt or killed ( $n=548 ; 38.36 \%$ ), and hurricane, flood, earthquake, tornado, or fire $(n=504 ; 33.7 \%)$.

\subsubsection{Midwestern university students}

A total of 1049 participants completed the questionnaires. Among potential subjects, 538 (52.3\%) did not endorse trauma exposure on the SLESQ and were therefore excluded from the primary analyses. In addition, 11 participants were excluded for endorsing their worst trauma whilst indicating it was only through electronic media and not connected to their occupation. Finally, 
three subjects were excluded for missing the majority of responses on all the measures; resulting in an effective sample size of 497. The remaining missing data was estimated in Mplus 7.0 using weighted least squares estimation with a mean- and variance-adjusted chisquare (WLSMV).

\subsubsection{Effective sample characteristics}

The effective sample $(n=497)$ had a mean age of $20.1(S D=4.5)$, which ranged from 18 to 56 years old. The majority of subjects were female ( $n=364,72.8 \%$ ). Most were employed part-time ( $n=245$, $49.0 \%)$, unemployed students $(n=162,32.4 \%)$ and employed fulltime $(n=50,10.0 \%)$. Participants primarily identified as single ( $n=399,79.8 \%)$, living with a significant other ( $n=78,15.6 \%)$, married ( $n=15,3.0 \%$ ), or divorced, separated or widowed $(n=6$, $1.2 \%)$. Most were Caucasian ( $n=375,75 \%)$, African-American ( $n=109,21.8 \%)$, Hispanic or Latino $(n=34,6.8 \%)$ or Asian ( $n=21,4.2 \%)$.

In the study sample, the most endorsed traumatic events were "family member or close friend died of accident, homicide or suicide" ( $n=231,46.5 \%)$, "physically harmed by someone ( $n=159$, $32.0 \%)$ ", and "serious car accident" ( $n=148,29.8 \%)$. The final question prompts respondents to elect their most distressing trauma to bear in mind when answering items on the subsequent questionnaire. The most commonly reported most distressing traumas were "family member or close friend died", $(n=228$, $45.9 \%)$, "adolescent sexual abuse" ( $n=37,7.4 \%$ ), and "physically forced to have sex" ( $n=35,6.8 \%)$.

\subsubsection{Data analysis}

Six CFAs were conducted in each sample using Mplus 7.1 software (see Table 1). PTSD items were treated as ordinal data, using a polychoric covariance matrix and probit coefficients for factor loadings. Weighted least squares estimation with a mean- and variance-adjusted chi-square (WLSMV) was used to estimate the models, which is preferred for estimating ordinal data (Flora and Curran, 2004; Wirth and Edwards, 2007). Error covariances and factor variances were fixed to zero and one, respectively, to scale the factors within the specified models. Chi-square difference tests were conducted to compare the nested models using a correction factor (Muthén and Muthén, 2010). Chi-square difference testing is not possible with non-nested models however, and as such, Bayesian Information Criterion (BIC) values were derived from ML estimation to compare non-nested models. In comparing BIC values, a difference of 10 points represents strong support that the model with the lower BIC value fits best (Kass and Raftery, 1995).

The following goodness of fit indices were obtained for each of the specified models: the comparative fit index (CFI), Tucker Lewis index (TLI), and the root mean square error of approximation (RMSEA). According to the benchmarks proposed by Hu and Bentler (1999), models fitting very well (and adequate) are indicated by CFI and TLI $\geq .95(.90-.94)$ and RMSEA $\leq .06(.07-.08)$.

\section{Results}

Based on DSM-5 diagnostic criteria in the NHRVS veteran sample, 143 (weighted $=12.0 \%$ ) screened positive for lifetime PTSD and 64 (weighted $=5.2 \%$ ) for past-month PTSD. In the Midwestern sample $4.02 \%$ of the undergraduate sample would meet the criteria for a probable PTSD diagnosis.

\subsection{U.S. veterans}

In the NHVRS sample, both the 6-factor Externalizing Behaviors and Anhedonia models provided a significantly better fit than the 4-factor DSM-5 model $\left(\Delta \chi^{2}(9)=63.06, p<.001 ; \Delta \chi^{2}(9)=181.88\right.$, $p<.001$, respectively) the 4-factor Dysphoria model $\left(\Delta \chi^{2}(9)=43.56, p<.001 ; \Delta \chi^{2}(9)=158.37, p<.001\right)$ and the 5factor Dysphoric Arousal model $\left(\Delta \chi^{2}(5)=33.06, p<.001\right.$; $\chi^{2}(5)=131.80, p<.001$, respectively). Comparing the two 4 -factor models, which are non-nested, the 4-factor Dysphoria model provided a better fit than the 4-factor DSM-5 model, as evidenced by a 56.15-point BIC difference. Comparing the two 6-factor models (which are non-nested), the Anhedonia model provided a better fit than the Externalizing Behaviors model, as evidenced by a 441.18point BIC difference. The 7-factor hybrid model provided a significantly better fit than all other models, including the 4-factor DSM-5 model $\left(\Delta \chi^{2}(15)=217.09, p<.001\right)$, the 4 -factor Dysphoria model $\left(\Delta \chi^{2}(15)=196.15, p<.001\right)$, the 5 -factor Dysphoric Arousal model $\left(\Delta \chi^{2}(11)=177.08, p<.001\right)$, the 6-factor Externalizing Behaviors model $\left(\Delta \chi^{2}(6)=137.33, p<.001\right)$, and the 6 -factor Anhedonia model $\left(\Delta \chi^{2}(6)=37.95, p<.001\right)$.

\subsection{Midwestern university students}

In the student sample, both the six-factor Externalizing Behaviors and Anhedonia models provided a significantly better fit than the 4-factor DSM-5 model $\Delta \chi^{2}(9)=123.76, p<.001$ $\Delta \chi^{2}(9)=191.91, p<.001$, respectively), 4-factor Dysphoria model $\left(\Delta \chi^{2}(15)=129.84, p<.001 ; \Delta \chi^{2}(15)=187.41, p<.001\right.$, respectively $)$ and the 5-factor Dysphoric Arousal model $\left(\Delta \chi^{2}(5)=54.43\right.$, $p<.001 ; \Delta \chi^{2}(5)=123.07, p<.001$, respectively). Comparing the two 4-factor models, which are non-nested, the DSM-5 model provided a better fit than the Dysphoria model, as evidenced by a 19.87-point BIC difference. Comparing the two 6-factor models (which are non-nested), the Anhedonia model provided a better fit than the Externalizing Behaviors model as evidenced by a 122.80 point BIC difference. The 7-factor Hybrid model provided a significantly better fit than all other models, including the 4-factor DSM5 model $\left(\Delta \chi^{2}(15)=246.96, p<.001\right.$.), the 4 -factor Dysphoria model $\left(\Delta \chi^{2}(15)=247.56, p<.001\right)$, the 5-factor Dysphoric Arousal model $\left(\Delta \chi^{2}(11)=181.98, p<.001\right)$, the 6-factor Externalizing Behaviors model $(\Delta \chi 2(6)=128.714, p<.001)$, and the 6-factor Anhedonia model $(\Delta \chi 2(6)=54.27, p<.001)$.

Table 2 reports the fit indices of competing models within both samples. Table 3 reports the standardized factor loadings across both samples; all were positive and statistically significant. Table 4 reports the inter-factor correlations for the veteran and student samples; all factors were highly correlated across both samples.

\section{Discussion}

The current study assessed six distinct DSM-5 PTSD models comprising between 4- and 7-factors of PTSD, across two diverse samples of veterans and university students. Our newly proposed 7-factor hybrid model was deemed to provide superior fit to both samples compared to all the other models. The 7-factor hybrid model was derived from theoretical and empirical evidence and consolidates previous work supporting findings that hyperarousal symptoms consist of anxious and dysphoric arousal (Elhai et al., 2011); that increased negative and reduced positive affect are two distinct constructs (Watson, 2005, 2009; Watson et al., 2011); and that externalizing behavior is distinct from other PTSD symptoms representing internal behaviors (Friedman, 2013; Steiner et al., 1997). All factor loadings were statistically significant positive, and high with the exception of the factor loading representing the trauma-related amnesia item. This has however been the case in several DSM-IV-TR based factor analytic studies (Armour and Shevlin, 2010). Given the different composition of the two samples, it is notable that the factor loadings of each item were similar across the two samples. The only deviation from this pertains to the 
Table 2

Confirmatory factor analyses fit indices for five PTSD models.

\begin{tabular}{lllllll}
\hline Model & $\chi^{2}$ & df & CFI & TLI & RMSEA $(90 \%$ CI $)$ & BIC \\
\hline Veterans & & & & & & \\
1 & 626.79 & 164 & .93 & .91 & $.044(.040-.047)$ & 50620.98 \\
2 & 606.80 & 164 & .93 & .92 & $.043(.039-.046)$ & 50564.83 \\
3 & 598.10 & 160 & .94 & .92 & $.043(.039-.047)$ & 50567.38 \\
4 & 562.00 & 155 & .94 & .92 & $.042(.038-.046)$ & 50483.96 \\
5 & 418.04 & 155 & .96 & .95 & $.034(.030-.038)$ & 50042.78 \\
6 & 374.41 & 149 & .96 & .95 & $.032(.028-.036)$ & 49945.92 \\
Midwestern students & & & & & \\
1 & 766.049 & 164 & .97 & .964 & $.086(.080-.092)$ & 27115.39 \\
2 & 782.985 & 164 & .96 & .963 & $.087(.081-.093)$ & 27135.26 \\
3 & 671.000 & 160 & .97 & .969 & $.080(.074-.087)$ & 27045.46 \\
4 & 614.857 & 155 & .98 & .971 & $.077(.071-.084)$ & 26988.37 \\
5 & 453.711 & 155 & .99 & .981 & $.062(.056-.069)$ & 26865.56 \\
6 & 383.550 & 149 & .99 & .985 & $.056(.049-.064)$ & 26817.58 \\
\hline
\end{tabular}

Note. Model $1=D S M-5$ emotional numbing; Model 2 = Dysphoria; Model 3 = Dysphoric arousal; Model $4=$ Externalizing; Model $5=$ Anhedonia; Model $6=$ Anhedonia/DA hybrid; $\chi^{2}=$ chi square; $\mathrm{df}=$ degrees of freedom; $\mathrm{CFI}=$ Comparative Fit Index; TLI = Tucker Lewis Index; RMSEA = Root Mean Square Error of Approximation; BIC = Bayesian Information Criterion.

item 'sleeping difficulty,' which appeared to be a clearer indicator of the dysphoric arousal factor in the veteran sample (see Table 3).

Elucidating the dimensional structure of PTSD is an ongoing research endeavor. Throughout the course of the DSM-IV (APA, 1994) and the DSM-IV-TR (APA, 2000), debates surrounding PTSD tripartite latent structure of PTSD commenced with the proposal of an alternative four-factor Emotional Numbing model (King et al., 1998), which gained prominence over a period of four years until the proposal of an alternative four-factor Dysphoria model (Simms et al., 2002). For the subsequent eight years, debates centered on which of these two models best represented the dimensional structure of PTSD (Yufik and Simms, 2010). In 2011, however, a fivefactor Dysphoric Arousal model was proposed and garnered support when assessed in comparison to the two four-factor models (Elhai et al., 2011).

The fifth edition of the DSM (DSM-5; APA, 2014) made several changes to PTSD's nosology, including the adoption of a 4-factor model that closely resembles the Emotional numbing model. In

Table 3

Factor loadings for the hybrid 7-factor model.

\begin{tabular}{|c|c|c|c|}
\hline \multirow[t]{2}{*}{ Symptom } & \multirow[t]{2}{*}{ Factor } & \multirow{2}{*}{$\begin{array}{l}\begin{array}{l}\text { Veteran } \\
\text { sample }\end{array} \\
\text { Factor } \\
\text { loadings }\end{array}$} & \multirow{2}{*}{$\begin{array}{l}\begin{array}{l}\text { Student } \\
\text { sample }\end{array} \\
\text { Factor } \\
\text { loadings }\end{array}$} \\
\hline & & & \\
\hline 1. Intrusive thoughts & $\mathrm{R}$ & .819 & .826 \\
\hline 2. Nightmares & $\mathrm{R}$ & .745 & .773 \\
\hline 3. Flashbacks & $\mathrm{R}$ & .733 & .774 \\
\hline 4. Emotional cue reactivity & $\mathrm{R}$ & .811 & .827 \\
\hline 5. Physiological cue reactivity & $\mathrm{R}$ & .763 & .817 \\
\hline 6. Avoidance of thoughts & A & .853 & .781 \\
\hline 7. Avoidance of reminders & A & .892 & .839 \\
\hline 8. Trauma-related amnesia & NA & .393 & .484 \\
\hline 9. Negative beliefs & NA & .817 & .781 \\
\hline 10. Blame of self or others & NA & .771 & .811 \\
\hline 11. Negative trauma-related emotions & NA & .875 & .904 \\
\hline 12. Loss of interest & An & .813 & .813 \\
\hline 13. Detachment & An & .900 & .866 \\
\hline 14. Restricted affect & An & .869 & .832 \\
\hline 15. Irritability/anger & EB & .855 & .839 \\
\hline 16. Self-destructive/reckless behavior & EB & .716 & .604 \\
\hline 17. Hypervigilance & AA & .829 & .727 \\
\hline 18. Exaggerated startle response & $\mathrm{AA}$ & .882 & .790 \\
\hline 19. Difficulty concentrating & DA & .892 & .842 \\
\hline 20. Sleep disturbance & DA & .803 & .672 \\
\hline
\end{tabular}

Note. $\mathrm{R}=$ reexperiencing; $\mathrm{A}=$ avoidance; $\mathrm{NA}=$ negative affect; $\mathrm{An}=$ anhedonia; $\mathrm{EB}=$ externalizing behaviors; $\mathrm{AA}=$ anxious arousal; $\mathrm{DA}=$ dysphoric arousal.
Table 4

Correlations among factors in both samples.

\begin{tabular}{llllllll}
\hline & $\mathrm{R}$ & $\mathrm{A}$ & $\mathrm{NA}$ & $\mathrm{An}$ & $\mathrm{EB}$ & $\mathrm{AA}$ & $\mathrm{DA}$ \\
\hline $\mathrm{R}$ & - & .876 & .824 & .732 & .781 & .775 & .750 \\
$\mathrm{~A}$ & .831 & - & .815 & .799 & .799 & .828 & .736 \\
$\mathrm{NA}$ & .762 & .721 & - & .815 & .830 & .780 & .792 \\
An & .685 & .601 & .840 & - & .843 & .838 & .924 \\
EB & .687 & .588 & .746 & .873 & - & .814 & .794 \\
AA & .677 & .606 & .722 & .701 & .740 & - & .862 \\
DA & .747 & .667 & .714 & .804 & .802 & .797 & - \\
\hline
\end{tabular}

Note. The top diagonal correlations are from the veteran sample and the lower diagonal correlations are from the student sample. $\mathrm{R}=$ reexperiencing; $\mathrm{A}=$ avoidance; $\mathrm{NA}=$ negative affect; $\mathrm{An}=$ anhedonia; $\mathrm{EB}=$ externalizing behaviors; $\mathrm{AA}=$ anxious arousal; $\mathrm{DA}=$ dysphoric arousal.

the year after the publication of DSM-5, researchers have provided evidence for the superior fit of two separate and distinct 6-factor DSM-5 models; the Anhedonia model (Liu et al., 2014) and the Externalizing Behaviors model (Tsai et al., in press). Although CFA studies of DSM-5 PTSD symptoms are in their infancy, this is reminiscent of the debate surrounding the two 4-factor Emotional Numbing and Dysphoria models. It is indeed likely that several studies will now compare these models to each other across a variety of trauma samples. Again reminiscent of earlier debates, and the proposal of the Dysphoric Arousal model, the 7-factor hybrid model consolidates key features of both 6 -factor models, which may be attributable to improved fit.

This current study aligns with prior research of Elhai et al. (2011), Liu et al. (2014), and Tsai et al. (in press) in that it supports each of the proposed changes to PTSD's latent structure; however, it suggests that all of these changes could be implemented into a single, theoretically- and empirically-grounded 7factor hybrid model. The separation of hyperarousal into dysphoric and anxious arousal was based on theoretical (Watson, 2005) and empirical evidence (Shevlin et al., 2009), reviewed in Elhai et al. (2011), and subsequently supported by several factor analytic studies (Armour et al., 2013a; Contractor et al., 2013; Harpaz-Rotem et al., 2014; Pietrzak et al., 2012; Reddy et al., 2013; Wang et al., 2013). Of note, these changes and subsequent support were based on hyperarousal as characterized in the DSMIV-TR (APA, 2000). In the 7-factor model conceptualization, anger/ irritability no longer loads on the dysphoric arousal factor; rather dysphoric arousal is indicated by the items of difficulty concentrating and sleep disturbance, whereas anxious arousal is indicated by hypervigilance and exaggerated startle response (see Table 1).

The irritability/anger item joins an item of self-destructive/ reckless behavior, which is categorized as a new externalizing behaviors factor. This was the main change implemented by Tsai et al. (in press). The creation of this factor was based on a body of literature proposing that individuals suffering from PTSD often display aggression, recklessness, and suicidality (Friedman, 2013), which may be representative of difficulties with impulse control and emotion regulation (Steiner et al., 1997) and distinct from other PTSD items representing behaviors which are more internally based such as trauma-related thoughts and feelings.

Liu et al. (2014) based their separation of the NACM factor on the premise that the factor comprises symptoms of both reduced positive affect and enhanced negative affect, which are theoretically and empirically proposed as two distinct constructs (Watson, 2005, 2009; Watson et al., 2011). In addition, the newly proposed National Institute of Mental Health Research Domain (RDoC) project differentiates between positive and negative valence systems (Cuthbert and Kozak, 2013), which further supports the separation of the NACM factor. 
Ultimately, elucidating the dimensional structure of PTSD has several clinical implications. First, the latent structure implemented by the DSM has implications for diagnostic algorithms, prevalence rates (Elhai et al., 2009), and thus individual diagnostic status of many individuals; for many, diagnostic status can be the gateway to services and support mechanisms. Second, identifying the optimal dimensional representation of PTSD symptoms can help inform the knowledge base in relation to co-morbidity between PTSD and other psychiatric morbidities (Elhai and Palmieri, 2011), as well as etiologic models of this disorder. Third, correctly identifying PTSD symptom groupings allows researchers to investigate if one particular grouping is more or less related to the ongoing maintenance of posttraumatic stress; leading further to the targeting of particular symptom groupings in treatment planning (Schell et al., 2004; Pietrzak et al., 2013a).

In summary, understanding that there may be 7 symptom clusters of PTSD may help to further refine models of the dimensional structure of PTSD symptoms in both research and clinical settings. For example, if dysphoric arousal is indeed a sub-construct within PTSD, that is more specific than a general dysphoria factor, this could be helpful in understanding the nature of the psychopathology of this sub-construct. Furthermore, it helps understanding in relation to how the symptoms develop and whether these symptoms require different interventions to alleviate them compared to alternative PTSD symptoms clusters. Notably, a change to diagnostic criteria and to the latent structure of a disorder has implications for diagnostic algorithms. For example, moving from DSM-IV-TR criteria to DSM-5 criteria has been shown to result in a high proportion of soldiers who previously met the diagnostic criteria no longer meeting new diagnostic criteria (Hoge, Riviere, Wilk, Herrel \& Weathers, 2014). Therefore, further refinement of the latent structure may result in greater discordance in past and present PTSD status. This has a range of implications related to compensation and policy. Therefore, careful considerations must be made in relation to decreased heterogeneity in symptom presentation vs. treatment provision.

Methodological limitations pertain to both of the samples utilized within the current study. First, each was assessed for PTSD using a self-report measure. It is possible that PTSD's latent structure may differ if PTSD is assessed via a clinical interview, or indeed alternative self-report measures. Therefore, future studies should assess the 7-factor hybrid model across a wide variety of assessment instruments. Second, as with many other PTSD CFA studies, results of the current study may be limited by order effects. Indeed, the order of items presented in both samples was identical. Marshall, Schell, and Miles, (2013) state that what “... researchers may be interpreting as clinically meaningful certain features of PTSD data, might be better attributed to specific measurement processes." (p. 63). Third, we did not assess the differential relations between external measures of psychopathology, such as depression, as it was felt that this was beyond the scope and primary focus of this paper. Fourth, general methodological issues also pertain to the 7-factor model itself, most notably that it is comprised of four latent factors that have only two indicators. Kline (2005) proposed that latent variables should consist of a minimum of three manifest indicators; however, to date, several prominent models of the dimensional structure of PTSD have included latent factors indicated by two items; including the current DSM-5 model and its avoidance factor (APA, 2014). Fifth, because both samples were cross-sectional, we were unable to extend the analyses to evaluate whether a particular factor of the hybrid model drives the course of PTSD or indeed if the hybrid model displays temporal stability. Recent research by Keane et al. (2014) assessed and supported the temporal stability of the DSM-5 four-factor model. This is a potential line of enquiry that could be examined in future work as it relates to both the Anhedonia and Externalizing models, and the newly proposed seven-factor Hybrid model. Sixth, we acknowledge that the Dysphoria model and the Dysphoric Arousal model in the current study are DSM-5 based conceptualizations and therefore include items which were not part of the DSM-IV-TR PTSD nosology, and thus the original conceptualization of these models. For example, the self-destructive/reckless behavior symptom was an indicator of the DA factor in the Dysphoric Arousal model rather than within the AA factor. It could however be argued that it would have been just as appropriate to place it in the AA factor based on literature on emotional dysregulation and impulsivity suggesting that impulsive self-destructive behaviors may be related to anxiety or depression (Weiss, Tull, Anestis and Gratz, 2013).

Further research will be useful in attempting to replicate the findings reported herein; and evaluating the external validity of the seven-factor model in relation to measures of comorbid psychopathology and biomarkers implicated in PTSD, prognostic utility of this model, and differential sensitivity of the seven factors in assessing PTSD treatment response.

\section{Role of the funding source}

The funding source had no role in study design; in the collection, analysis and interpretation of data; in the writing of the report; or in the decision to submit the paper for publication.

\section{Contributors}

CA conducted the literature review, conceptualized the study design, assisted with the analysis, wrote the first draft of the manuscript, and handled subsequent drafts after receiving coauthors feedback.

JT conducted the veteran analysis and commented on drafts.

TD conducted the student analysis and commented on drafts.

$\mathrm{RC}$ assisted with the student analysis and commented on drafts.

TB collected the student data and commented on drafts.

JE assisted with the analysis and commented on drafts.

RP collected the veteran data, conceptualized the study design, and commented on drafts.

\section{Conflict of interest}

Dr. Pietrzak is a scientific consultant to Cogstate, Ltd. for work that bears no relationship to the present study. None of the other authors report any conflicts of interest.

\section{Acknowledgments}

The National Health and Resilience in Veterans Study was funded by the U.S. Department of Veterans Affairs National Center for Posttraumatic Stress Disorder (PTSD). Preparation of this report was supported in part by the Clinical Neurosciences Division of the National Center for PTSD and a private donation. The views and opinions expressed in this report are those of the authors and should not be construed to represent the views of sponsoring organizations, agencies, or the U.S. government.

\section{References}

American Psychiatric Association. Diagnostic and statistical manual of mental disorders. 4th ed. Washington; DC: American Psychiatric Association; 1994.

American Psychiatric Association. Diagnostic and statistical manual of mental disorders. 4th text revised ed. Washington; DC: American Psychiatric Association; 2000.

American Psychiatric Association. Diagnostic and statistical manual of mental disorders. 5th ed. Washington; DC: American Psychiatric Association; 2014. 
Armour C, Contractor AA, Palmieri PA, Elhai JD. Assessing latent level associations between PTSD and dissociative factors: is depersonalization and derealization related to PTSD factors more so than alternative dissociative factors? Psychol Inj Law 2014;7:131-42.

Armour C, Carragher N, Elhai JD. Assessing the fit of the dysphoric arousal model across two nationally representative epidemiological surveys; the Australian NSMHWB and the United States NESARC. J Anxiety Disord 2013a;27:109-15.

Armour C, Elhai JD, Richardson D, Ractliffe K, Wang L, Elklit A. Assessing a five factor model of PTSD: is dysphoric arousal a unique PTSD construct showing differential relationships with anxiety and depression? J Anxiety Disord 2012;26:368-76.

Armour C, Ghazali SR, Elklit A. PTSD's latent structure in Malayan tsunami victims: assessing the newly proposed dysphoric arousal model. Psychiat Res 2013b;1: $26-32$.

Armour C, Shevlin M. Testing the dimensionality of PTSD and the specificity of the dysphoria factor. J Loss Trauma 2010;15:11-27.

Asmundson GJG, Stapleton JA, Taylor S. Are avoidance and numbing distinct PTSD symptom clusters? J Trauma Stress 2004;17:467-75.

Biehn TL, Elhai JD, Seligman LD, Tamburrino M, Armour C, Forbes D. Underlying dimensions of DSM-5 posttraumatic stress disorder and major depressive disorder symptoms. Psychol Inj Law 2013;6:290-8.

Carlson EB, Smith SR, Palmieri PA, Dalenberg C, Ruzek JI, Kimerling R, et al. Development and validation of a brief self-report measure of trauma exposure: the trauma history screen. Psychol Assess 2011;23:463-77.

Contractor AA, Durham TA, Brennan JA, Armour C, Wutrick HR, Frueh CB, et al. DSM-5 PTSD's symptom dimensions and relations with major depression's symptom dimensions in a primary care sample. Psychiat Res 2014;215:146-53.

Contractor AA, Layne CM, Steinberg AM, Ostrowski SA, Ford JD, Elhai JD. Do gender and age moderate the symptom structure of PTSD? Findings from a nationa clinical sample of children and adolescents. Psychiat Res 2013;210:1056-64.

Cuthbert BN, Kozak MJ. Constructing constructs for psychopathology: the NIMH research domain criteria. J Abnorm Psychol 2013;122:928-37.

Elhai JD, Biehn TL, Armour C, Klopper JJ, Frueh BC, Palmieri PA. Evidence for a unique PTSD construct represented by PTSD's D1-D3 symptoms. J Anxiety Disord 2011:25:340-5.

Elhai JD, Ford JD, Ruggiero KJ, Frueh BC. Diagnostic alterations for posttraumatic stress disorder: examining data from the National Comorbidity Survey Replication and National Survey of Adolescents. Psychol Med 2009;39:1957-66.

Elhai JD, Franklin CL, Gray MJ. The SCID PTSD Module's trauma screen: validity with two samples in detecting trauma history. Depress Anxiety 2008;25:737-41.

Elhai JD, Miller ME, Ford JD, Biehn TL, Palmieri PA, Frueh BC. Posttraumatic stress disorder in DSM-5: estimates of prevalence and symptom structure in a nonclinical sample of college students. J Anxiety Disord 2012;26:58-64.

Elhai JD, Palmieri PA. Posttraumatic stress disorder symptom instruments and factor structure: an update on the current literature and advancing a research agenda. J Anxiety Disord 2011;25:849-54.

First MB, Spitzer RL, Gibbon M, Williams JBW. Structured clinical interview for DSM-IV-TR Axis I disorders. New York: Biometrics Research, New York State Psychiatric Institute; 2002.

Flora DB, Curran PJ. An empirical evaluation of alternative methods of estimation for confirmatory factor analysis with ordinal data. Psychol Methods 2004;9:466-91.

Friedman MJ. Finalizing PTSD in DSM-5: getting here from there and where to go next. J Trauma Stress 2013;26:548-56.

Friedman MJ, Resick PA, Bryant RA, Brewin CR. Considering PTSD for DSM-5. Depress Anxiety 2011;28:750-69.

Goodman LA, Corcoran C, Turner K, Yuan N, Green BL. Assessing traumatic event exposure: general issues and preliminary findings for the Stressful Life Events Screening Questionnaire. J Trauma Stress 1998;11:521-42.

Gootzeit J, Markon K. Factors of PTSD: Differential specificity and external correlates. Clin Psychol Rev 2011;31:993-1003.

Harpaz-Rotem I, Tsai J, Pietrzak RH, Hoff S. The dimensional structure of posttraumatic stress symptomatology in 323,903 U.S. veterans. J Psychiat Res 2014;49:31-6.

Hoge CW, Riviere LA, Wilk J, Herrell RK, Weathers FW. The prevalence of posttraumatic stress disorder in US combat infantry soldiers: a head-to-head comparison of DSM-5 with DSM-IV-TR criteria screening checklists. Lancet Psychiatry 2014

Hu L, Bentler PM. Cutoff criteria for fit indexes in covariance structure analysis: Conventional criteria versus new alternatives. Struct Equa Mod: A Multidiscip 1999;6:1-55.

Kass RE, Raftery AE. Bayes factors. J Am Stat Assoc 1995;90:773-95.

Keane TM, Rubin A, Lachowicz M, Brief D, Enggasser JL, Roy M, et al. Tempora stability of DSM-5 posttraumatic stress disorder criteria in a problem-drinking sample. Psychol Assess 2014 (in press).
King D, Leskin G, King L, Weathers F. Confirmatory factor analysis of the ClinicianAdministered PTSD Scale: evidence for the dimensionality of posttraumatic stress disorder. Psychol Assess 1998;10:90-6.

Kline RB. Principles and practice of structural equation modeling. 2nd ed. New York: Guilford; 2005.

Liu P, Wang L, Cao C, Wang R, Zhang J, Zhang B, et al. The underlying dimensions of DSM-5 posttraumatic stress disorder symptoms in an epidemiological sample of Chinese earthquake survivours. J Anxiety Disord 2014;28:345-51.

Marshall GN, Schell TL, Miles J. A multi-sample confirmatory factor analysis of PTSD symptoms: what exactly is wrong with the DSM-IV structure? J Clin Psychol Rev 2013;33:54-66.

McDonald SD, Calhoun PS. The diagnostic accuracy of the PTSD checklist: a critical review. Clin Psychol Rev 2010;30:976-87.

Miller MW, Wolf EJ, Kilpatrick D, Resnick H, Marx BP, Holowka DW, et al. The prevalence and latent structure of proposed DSM-5 posttraumatic stress disorder symptoms in U.S. national and veteran samples. Psychol Trauma: Theory, Res Pract Policy 2013;5:501-12.

Muthén BO, Muthén LK. Technical appendices [Internet]. Los Angeles: Authors; 2010 [cited 2014 Jan 4]. Available from, http://www.statmodel.com/techappen. shtml.

Pietrzak RH, Tsai J, Harpaz-Rotem I, Whealin JM, Southwick SM. Support for a novel five-factor model of posttraumatic stress symptoms in three independent samples of Iraq/Afghanistan veterans: a confirmatory factor analytic study. J Psychiatr Res 2012;46:317-22.

Pietrzak RH, Feder A, Schechter CB, Singh R, Cancelmo L, Bromet EJ, et al. Dimensional structure and course of post-traumatic stress symptomatology in World Trade Center responders. Psychol Med 2013:2085-98.

Pietrzak RH, Galea S, Southwick SM, Gelernter J. Examining the relation between the serotonin transporter 5-HTTPLR genotype x trauma exposure interaction on a contemporary phenotypic model of posttraumatic stress symptomatology: a pilot study. J Affect Disord 2013b;148:123-8.

Reddy MK, Andersond BJ, Liebschutze J, Steind MD. Factor structure of PTSD symptoms in opioid-dependent patients rating their overall trauma history. Drug Alcohol Depend 2013;132:597-602.

Roberton T, Daffern M, Bucks RS. Emotion regulation and aggression. Aggress Violent Behav 2012;17:72-82.

Schell TL, Marshall GN, Jaycox LH. All symptoms are not created equal: the prominent role of hyperarousal in the natural course of posttraumatic psychological distress. J Abnorm Psychol 2004;113:189-97.

Shevlin M, McBride O, Armour C, Adamson G. Reconciling the differences between the King et al. (1998) and Simms, et al. (2002) factor models of PTSD. J Anxiety Disord 2009;23:995-1001.

Simms LJ, Watson D, Doebbeling BN. Confirmatory factor analyses of posttraumatic stress symptoms in deployed and non-deployed veterans of the Gulf War. J Abnorm Psychol 2002;111:637-47.

Steiner H, Garcia IV, Matthews Z. Posttraumatic stress disorder in incarcerated juvenile delinquents. J Am Acad Child Adolesc Psychiatry 1997;36:357-65.

Tsai J, Harpaz-Rotem I, Armour C, Southwick SM, Krystal JH, Pietrzak RH. Dimensional structure of DSM-5 posttraumatic stress symptoms: results from the National Health and Resilience in Veterans Study. J Clin Psychiatry 2014 (in press).

US Census Bureau. USA: current population survey [Internet]. 2010. c2010 - [cited 2014 Jan 4]. Available from, http://www.census.gov/cps/.

Wang M, Armour C, Li X, Dai X, Zu X, Yao S. Further evidence for a five-factor model of PTSD: factorial invariance across gender in Chinese earthquake survivors. J Nerv Ment Dis 2013;201:145-52.

Watson D. Rethinking the mood and anxiety disorders: a quantitative hierarchical model for DSM-V. J Abnorm Psychol 2005;114:522-36.

Watson D. Differentiating the mood and anxiety disorders: a quadripartite model. Annu Rev Clin Psychol 2009;5:221-47.

Watson D, Clark LA, Stasik SM. Emotions and the emotional disorders: a quantitative hierarchical perspective. Int J Clin Health Psychol 2011;11:429-42.

Weathers FW, Litz BT, Keane TM, Palmieri PA, Marx BP, Schnurr PP. Washington DC: the PTSD checklist for DSM-5 (PCL-5); c2014 [Internet]. 2013 [cited 2014 Jan 4]. Available from, www.ptsd.va.gov.

Weiss NH, Tull MT, Anestis MD, Gratz KL. The relative and unique contributions of emotion dysregulation and impulsivity to post-traumatic stress disorder among substance dependent inpatients. Drug Alcohol Depend 2013;128:45-51.

Wirth RJ, Edwards MC. Item factor analysis: current approaches and future directions. Psychol Method 2007;12:58-79.

Yufik T, Simms LJ. A meta-analytic investigation of the structure of posttraumatic stress disorder symptoms. J Abnorm Psychol 2010;119:764-76. 